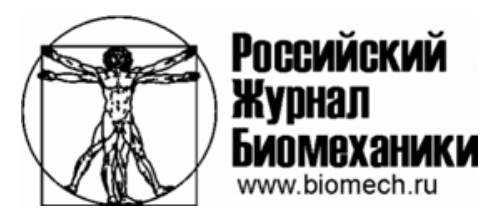

\title{
ТЕОРЕТИЧЕСКАЯ МОДЕЛЬ ПЕРЕСТРОЙКИ КОСТНОЙ ТКАНИ
}

\author{
А. Нутини \\ Центр по изучению науки о движении, Италия, 55100, Лукка, 94 via di Tiglio, loc. Arancio, e-mail: \\ hoxgene68@gmail.com
}

\begin{abstract}
Аннотация. Перестройка является сложным процессом, существенным для фризиологического функционирования костной ткани. В этой статье сделана попытка создать теоретическую модель этого процесса, учитывающую как биомеханическую, так и биохимическую регуляцию. Следовательно, перестройка костной ткани реализует сложную сеть, которая, в свою очередь, стимулирует конформационные изменения, требуемые как для здоровья костной ткани, так и для процессов восстановления. После краткого анализа перестройки костной ткани с точки зрения физиологии рассматривается математическое развитие идеи Вито Вольтерра об экологических взаимоотношениях между популяциями хищников и жертв, приспособление благодаря строгой аналогии к динамике клеточных популяций, являющихся протагонистами в перестройке кости. Учитывая колебательный характер этого явления в среде костных морфогенетических единиц, модель завершается сильной корреляцией между геометрией морфологии кости (пористость и поверхностная морфология) и двумя упомянутыми выше регулированиями. В диффреренциальное уравнение, рассматривающее субпопуляции остеокластов и остеобластов, которые непосредственно не вмешиваются В процесс перестройки и самообновления (линии клетокпредшественников), вводится также новая переменная состояния. Таким образом, в предложенную модель входят три дифференциальных уравнения, описывающие состояние ткани (в ситуациях здоровья или патологии) и работу перестройки в действии.
\end{abstract}

Ключевые слова: теоретическая модель, перестройка, остеокласты, остеобласты, костная морфогенетическя единица, модель «хищник-жертва», теория Вито Вольтерра, костная ткань.

\section{ВВеДЕНИЕ}

Процесс перестройки костной ткани весьма сложен и труден для анализа, и, хотя он является фундаментальным для физиологии самой ткани, недостаточно сведений о поведении и в величинах, влияющих на явление в целом.

Важность создания модели такого рода биологического явления состоит в том, чтобы попытаться понять как нормальную жизнедеятельность ткани, так и изменения, вызванные различными дегенеративными заболеваниями, которые включают значительные структурные отклонения параметров, поддерживающих патологическое развитие.

Сама кость - это орган со своими метаболическими потребностями и биохимическими принципами, которые при необходимости регулируют внутреннюю связь между основными единицами, перестройку или восстановление.

(C) Нутини А., 2015

Нутини Алессандро, кинезиолог Центра по изучению науки о движении, Лукка, Италия 
Таким образом, поведение костной ткани аналогично поведению реального органа с его метаболическими потребностями и биохимическими принципами, которые регулируют внутреннюю связь между различными величинами для хорошей перестройки или восстановления.

Макроскопически структура костной ткани представлена в виде серии полосок или слоев, которые растут путем осаждения новой ткани, и эта цель достигается благодаря взаимодействию четырех активных мобильных клеточных элементов: костных клеток-предшественников, остеобластов, остеоцитов и остеокластов.

Костные клетки-предшественники получены из внутреннего слоя надкостницы и дифференцируются в остеобласты, функция которых состоит в производстве исходной некристаллизованной матрицы кости, называемой «остеоидом ткани». После выработки этой матрицы остеобласты становятся остеоцитами, клетками покоя, захваченными самой матрицей, которые, несмотря на очевидную неподвижность, имеют много физиологических функций. И наоборот, остеокласты имеют прародителей в костном мозге (вместе с моноцитами) и принадлежат к другой линии клеток из первых трех элементов. Их функция заключается в поглощении кости, и у них есть рецепторы для кальцитонина и для стимулирующего фактора, который высвобождается остеобластами под управлением паратиреоидного гормона.

Таким образом, предлагаемая модель основана на взаимодействии этих элементов и других основополагающих связей для сохранения той же ткани и ее обычных функций.

\section{ОБЩАЯ СТРУКТУРА ПЕРЕСТРОЙКИ КОСТНОЙ ТКАНИ}

У кости есть удивительный внутренний динамизм, проявляющийся и в своей деятельности, и с точки зрения анаболического/катаболического равновесия.

Такую интенсивную деятельность можно обобщить четырьмя основными явлениями для исследуемой ткани: ранней резорбцией кости, приростом в длину и толщину, преобразованием губчатой кости в компактную кость и перестройкой компактной кости.

Ранняя резорбция кости происходит за счет остеокластов и при наличии хорошей сосудистой структуры; она достигается в примитивах трабекул путем возникновения остеобластов и имеет целью создание пространства для аппозиции новой ткани.

Прирост длины и толщины вместе с тем происходит благодаря пролиферации хондроцитов в ядра оссификации и, в частности, во время роста толщины кость формируется в процессе внутримембранной оссификации (аппозиции надкостницы), и одновременно остеокласты резорбируют костную ткань на эндосте, поддерживая структурную динамику, подходящую для полости костного мозга.

При превращении губчатой кости в компактную кость происходит постепенное преобразование костных трабекул в слоистые структуры, в то время как поверхность покрыта костными каналами с внутренними продольными сосудистыми структурами. Деятельность остеокластов становится более интенсивной, и внутри структуры роется большой канал, так называемый «туннель эрозии», в него в какой-то момент его формирования вторгаются костные клетки-предшественники, которые после дифференцирования в остеобласты начинают с производства матрицы и формирования компактной структуры (остеона).

Перестройка компактной кости - это необходимый ответ на механические стимулы, действующие на костную структуру; она делается одноядерными клетками, которые разъедают компактную кость и полностью разрушают различные Гаверсовы системы, позволяя последующее отложение новых остеонов. Ламеллы координированно удаляются как со стороны надкостницы, так и со стороны эндоста, 
чтобы образовать конечную структуру (и, следовательно, функциональную архитектуру) перестроенного костного сегмента. Перестройка кости работает не только как операция механической и функциональной реструктуризации, но и как перегруппировка деятельности минералов самого костного вещества, осуществляя биохимический кругооборот, что устанавливает минеральный гомеостаз.

Рассмотрим внимательно процесс перестройки, для которого автор хочет получить математическую модель. Согласно анализу Parfitt [11], перестройка происходит в несколько этапов:

1. Покой: на этой стадии поверхность кости покрыта плоскими клетками, так называемыми выстилающими клетками, возникающими из остеобластов: между ними и костной тканью вставляется остеоидная ткань. Эти плоские клетки имеют рецепторы для различных веществ, стимулирующих поглощение костной ткани, что вызывает поверхностную резорбцию остеоидной ткани: таким образом объясняется минеральная часть.

2. Активация: начинается набор предшественников остеокластов, которые приносятся кровью через каналы Фолькмана и Гаверса; прибыв, они прикрепляются к поверхности кости путем процесса адгезии при помощи специфических белков (остеопонтина, остеокальцина и т.д.).

3. Резорбция: на этой фазе основную роль играют остеокласты, абсорбирующие костную ткань.

4. Реверсирование: это интервал времени между завершением резорбции и началом формирования ткани. Остеобласты привлекаются в пространство резорбции химическими раздражителями с последующей адгезией, пролиферацией и дифференцировкой постепенным и скоординированным образом, так формируется новая ткань. Одновременно с этим появляются фагоциты, имеющие, похоже, особую роль: сгладить оставленные резорбцией неровности.

5. Формирование: сначала остеоидная ткань синтезируется и откладывается в определенных местах, а дальнейшей минерализацией управляют остеобласты.

Набор всех этих функций происходит на уровне так называемых «костных морфогенетических единиц», которые в соответствии с их числом, состоянием активации и позиционированием характеризуют перестройку костной ткани саму по себе. Как легко видеть, все эти события можно вкратце описать как весьма плотную и сложную информационную сеть, из которой кажется невозможным извлечь точную картину.

Цель данной работы состоит в создании модели, на которой можно проверить физиологию исследуемой ткани и продемонстрировать те события, которые характеризуют ее жизнь, либо функциональные изменения, которые могут развиться вследствие патологии.

\section{МАТЕМАТИЧЕСКАЯ МОДЕЛЬ ПЕРЕСТРОЙКИ КОСТИ (I) - ОСНОВНЫЕ ПРИНЦИПЫ}

В качестве основного принципа в теоретическом обосновании модели костной ткани надо учитывать функциональное и динамическое взаимодействие остеобластов и остеокластов внутри костной морфогенетической единицы в течение самого процесса перестройки.

Хотя наличие остеоцитов, несомненно, необходимо для физико-химического функционирования ткани, в структурной динамике одного события перестройки оно вторично (по сравнению с остеобластами и остеокластами).

Имея в виду недавнюю работу P.G. Genever, R.J. Patton и M. Fagan [2], видимо, очень удобно использовать модель системы «хищник-жертва», т.е. конкурентную модель между ростом двух популяций, одна из которых жертва, а другая хищник, поскольку считается, что даже клеточная популяция не может уменьшаться до отрицательных значений. 
Однако применение этой системы, по мнению автора, зависит от некоторых ограничений, которые нуждаются в дальнейшем уточнении. Например, в модели «хищник-жертва» в отсутствие хищника жертва растет без мальтузианских ограничений. Это явление, по-видимому, не отражается во взаимоотношениях остеокластов или остеобластов; из-за сильной биохимической компоненты регулирования это допускает своего рода гомеостаз в производстве этих двух клеточных линий. В той же модели установлено, что популяция хищников уменьшает популяцию жертвы в соотношении, пропорциональном росту этих двух популяций: в костной ткани процесс перестройки не обеспечивает такую ситуацию во взаимодействии остеокластов и остеобластов, даже если можно определить общую картину. Наконец можно предположить, даже для костной ткани, экспоненциальный распад популяции хищников в отсутствие добычи, связывая это событие с явлением остеопороза, где имеется дисбаланс в функциональных особенностях между «производителями» и «разрушителями» костной ткани.

В исследуемом костном сегменте перестройка создает своего рода «структурную трофическую сеть», которая не обеспечивает функциональной и структурной однородности всего органического окружения, поскольку, например, минерализация матрицы происходит с нелинейной логикой и проявляет разные особенности в различных местах, где она наблюдается. Модель «хищник-жертва» учитывает периодическую систему, генератор, который не присутствует в кости в целом. Однако можно преодолеть эту трудность, если рассмотреть меньшую систему, такую как костная морфогенетическая единица, где развитие среды этого явления может считаться однородным и с колебательным поведением.

В целом, прежде чем продолжить, можно предположить некоторые факты:

- Взаимодействие между двумя популяциями клеток (остеоцитами и остеокластами) гарантируется даже из регуляторных и биохимических механизмов трансдукции и подразумевается в разрабатываемой в этой статье модели.

- Существует общая математическая модель для процесса перестройки кости, ставящая популяции остеобластов и остеокластов в функциональное отношение.

- В случае отсутствия какой-то линии клеток (например снижения активности остеобластов) есть экспоненциальный рост другой линии (например остеокластов) и наоборот (экспоненциальная редукция).

- Морфологические и функциональные механические модификации пропорциональны изменению клеточных популяций, способствующих формированию процесса перестройки.

Бесспорно, модель «хищник-жертва» оставляет некоторые открытые вопросы при применении к клеточным популяциям, вовлеченным в изучаемый процесс, но это хороший выбор, потому что объяснение сложного биологического явления слишком часто требует применения более сложных математических моделей, каждой со своими характеристиками и конкретными гипотезами, которые приводят к рассмотрению этого вопроса на нескольких уровнях, часто приходится анализировать систему с использованием различных динамических и математических предположений, что часто может даже отвлечь внимание от цели.

Чтобы адаптировать так называемый «принцип Вольтерра» [14] к явлению перестройки костной ткани и вывести отсюда модель, совместимую с физиологическим явлением не следует применять более или менее сложные модели с чрезвычайно громоздким анализом и малополезными заключениями. Стоит помнить, что Вито Вольтерра написал в своей знаменитой работе, включенной в «Записки Итальянского королевского талассографического комитета» [15], о сосуществования двух видов в одной и той же среде, со специальным намерением создать надежную математическую модель: 
«Чтобы справиться с проблемой математически, мы должны начать с гипотезы, даже далекой от реальности, которая даст приблизительную картину. Даже если гипотеза будет вначале грубой, или же слишком простой, ее можно применить для расчета и проверить количественно или качественно; если полученные результаты соответствуют статистическим данным, а затем проверить правильность предположений для статистического обоснования новых результатов. Поэтому лучше, с целью облегчить применение расчета, очертить явление, выделив действия, которые вы хотите изучить, полагая, что они функционируют самостоятельно, и игнорировать другие».

Эта информация является наиболее подходящей для рассмотрения возможной модели, которая проверяет явление перестройки костной ткани, соединяя воедино механическое развитие, вызванное самим процессом, с динамикой вовлеченных клеточных популяций.

\section{МАТЕМАТИЧЕСКАЯ МОДЕЛЬ ПЕРЕСТРОЙКИ КОСТИ (II) - РАЗВИТИЕ}

Развитие модели «хищник-жертва» Вито Вольтерра базируется в основном на двух дифференциальных уравнениях:

$$
\begin{gathered}
\frac{d S}{d t}=[\text { Рост популяции жертвы }]-\left[\text { Коэффициент захвата } \frac{\text { Жертва }}{\text { Хищник }}\right] P, \\
\frac{d P}{d t}=[\text { Число рождений хищников на захват }] P-[\text { Число смертей хищников }],
\end{gathered}
$$

где $S$ - размер популяции жертвы, а $P$ - то же для хищника. Эти уравнения привели к созданию различных моделей, учитывающих различные пути роста популяции жертвы: линейный, геометрический, экспоненциальный и т.д.

Очевидно, уравнения (1) и (2) становятся более сложными при рассмотрении взаимодействий с экологическими переменными (например наличие нескольких источников питания для хищника) или внезапных изменений в ключевых переменных (когда число жертв внезапно и резко увеличивается, создаются новые взаимодействия с экосистемой).

Следует отметить, что второй член в (1) также называется «функциональным откликом», в то время как первый член в (2) определяется как численный ответ, и эти параметры должны быть надлежащим образом приняты во внимание при рассмотрении биологических систем, которые соответствуют динамике системы «жертва-хищник». В этом случае окружение костной морфогенетической единицы считается постоянным таким образом, что взаимодействие двух вовлеченных клеточных популяций варьируется в ограниченном диапазоне, и сосуществование этих групп может рассматриваться как имеющее колебательный характер; т.е. популяции остеокластов и остеобластов до бесконечности колеблются из одной фазы в другую с точкой равновесия, в которой эти два типа клеток не флуктуируют, но с таким нестабильным характером, что даже небольшие изменения в популяции вызывают потерю этого равновесия и перенастройку колебательного поведения (а значит и при наличии постоянного окружения костной морфогенетической единицы небольшие изменения в популяции вызывают колебания). Характерные уравнения этой модели имеют следующий вид:

$$
\begin{gathered}
\frac{d S}{d t}=r S-(a S) P, \\
\frac{d P}{d t}=b(a S) P-m P,
\end{gathered}
$$


где $r$ - коэффициент роста популяции жертвы; $m$ - коэффициент смертей в популяции хищников; $a$ и $b$ - константы, указывающие на линейный отклик как на функциональный отклик в (3), заданный $(a S)$, так и численный ответ в (4), который задается величиной $b(a S)$ и конфигурируется как «функция от функции» (т.е. численный ответ как функция от функционального отклика). Эта система уравнений приводит к колебательному графику, как показано на рис. 1, и можно видеть, как увеличение популяции жертв соответствует увеличению популяции хищников, а затем как популяция жертв уменьшается (из-за непрерывной охоты хищников), то же происходит с хищниками и цикл повторяется.

Описанную модель «хищник-жертва» можно адаптировать к логике остеобластов и остеокластов костной ткани, принимая во внимание все шаги в направлении клеточной дифференциации этих двух клеточных линий, остеобластов и остеокластов (стартующих от соответствующих предшественников), которые включены в вариации клеточных популяций.

Заменяя $S$ на $X_{o s b}$ (популяцию остеобластов) и $P$ на $X_{o s c}$ (популяцию остеокластов) и думая о возможной функциональной аналогии, можно выразить изменения популяций как $d X_{o s b} / d t$ и $d X_{o s c} / d t$ соответственно.

В соответствии с работой P.G. Genever, R.J. Patton и M. Fagan [2], автор также считает полезным предложить модель типа «хищник-жертва», модифицированную G.F. Gause et al. [1], которая обеспечивает анализ динамики между остеобластами и остеокластами, не влияя на специфические биологические механизмы, лежащие в основе перестройки кости.

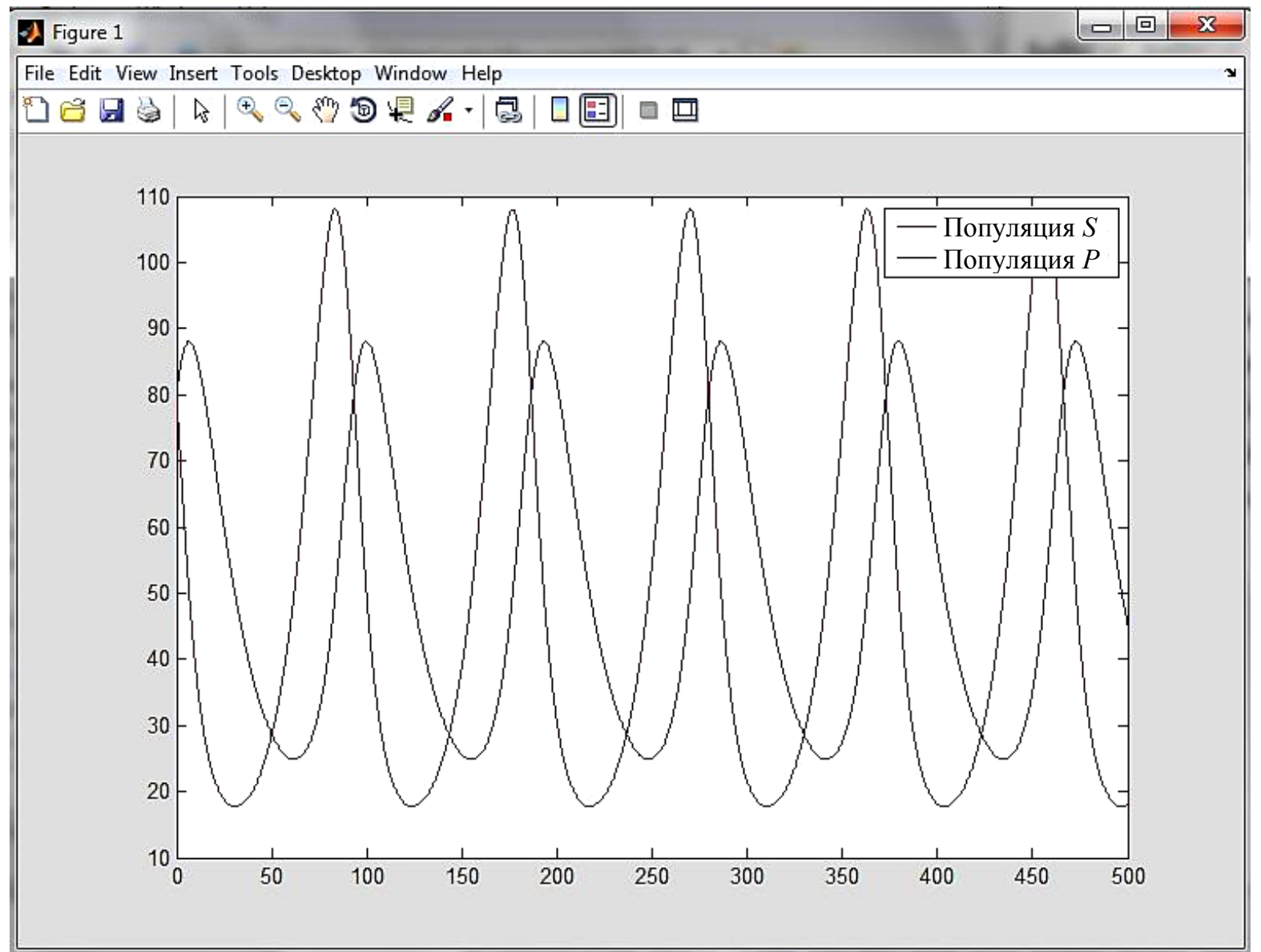

Рис. 1. Примеры колебательной модели Вольтерра-Лотке (графики получены в Matlab $^{\circledR}$ при установлении первоначальный непрерывности и значений параметров, описанных в тексте $(S$ - остеобласты, $P$ - остеокласты) 
С этой целью система уравнений принимает вид

$$
\begin{gathered}
\frac{d X_{o s b}}{d t}=a-b \sqrt{X_{o s b(t)}}, \\
\frac{d X_{o s c}}{d t}=c \frac{X_{o s c(t)}^{2}}{K_{o s c}+X_{o s c(t)}^{2}}-d,
\end{gathered}
$$

где $a, b, c, d, e, K_{o s c}$ - скалярные параметры.

Предложенная модель (все еще обнаруживающая и численный ответ, и функциональный отклик) дает решения для $X_{o s b}$ и $X_{o s c}$, осуществляемые в периодических орбитах для всех положительных условий типа $X_{o s c(0)}, X_{o s b(0)}>0$, кроме одной положительной точки равновесия. Представленная система гораздо ближе к реальной функциональности обработанных клеточных популяций, так как она выражает нелинейность, отображаемую внутренней динамикой костной морфогенетической единицы, и позволяет оценить всю систему из соотношения двух откликов (численного и функционального). Однако эта модель не включает в себя сигнал активации процесса (обычно биомеханической природы) и выражает периодичность, которая осуществляется не в единственном цикле, а в серии циклов перестройки.

Тем не менее в рассматриваемой системе остается скрытым вклад системы биохимической связи, что стоит в будущем включить в предложенную модель. Как уже показано ранее (в самых общих чертах), система самовосстановления и адаптации кости (перестройка) включает в себя участие костной морфогенетической единицы, которое вычисляется при их массовом присутствии, в нормальном взрослом человеческом скелете их около $1,7 \cdot 10^{6}[10]$. Это моделирование структурной сети, действительно, является активным и связывается благодаря некоторым сигналам, обеспечивающим определенный продуктивный гомеостаз между клеточными популяциями, составляющими сами костные морфогенетические единицы.

При возникновении расстройства этот гомеостаз утрачивается и образуется повреждение типа такого заболевания, как остеопороз, когда структура деградирует и, следовательно, такие важные механические свойства, как напряжения и прочность, значительно снижаются.

Функциональному балансу в костной морфогенетической единице способствуют два важных параметра: биохимическое регулирование и механическое регулирование, которые постоянно взаимодействуют друг с другом.

Биохимическое регулирование осуществляется через различные факторы, влияющие на весь процесс перестройки. Формирование остеокластов строго зависит от биохимических элементов, таких как фактор MCSF (фактор, стимулирующий колонии макрофагов), $R A N K$ (активатор рецепторов ядерного фактора каппа-B), RANK-Ligand и молекула $O P G$ (остеопротегерин).

Короче говоря, RANKL активирует рецептор $R A N K$ у предшественников остеокластов, который вызывает дальнейшую дифференциацию и поддерживает их деятельность, в то время как молекула $O P G$ (называемая также «рецептор-приманка») выступает в качестве ингибитора таких действий, так как она связывается с RANKL и предотвращает соединение с $R A N K$ [4] (см. рис. 2 и объяснение в подписи к нему).

Трансформирующий фактор роста $B T G F p$, точнее член надсемейства костных морфогенетических белков $B M P$, играет важную роль в коммуникации остеобластов и остеокластов в перестройке кости. Этот фактор накапливается в костной матрице и в этой среде оказывает свое действие на различные клетки при резорбции остеокластами. В качестве примера см. действие $B M P-3$ как ингибитора дифференцировки остеобластов и, следовательно, как негативного регулятора плотности кости [9]. 


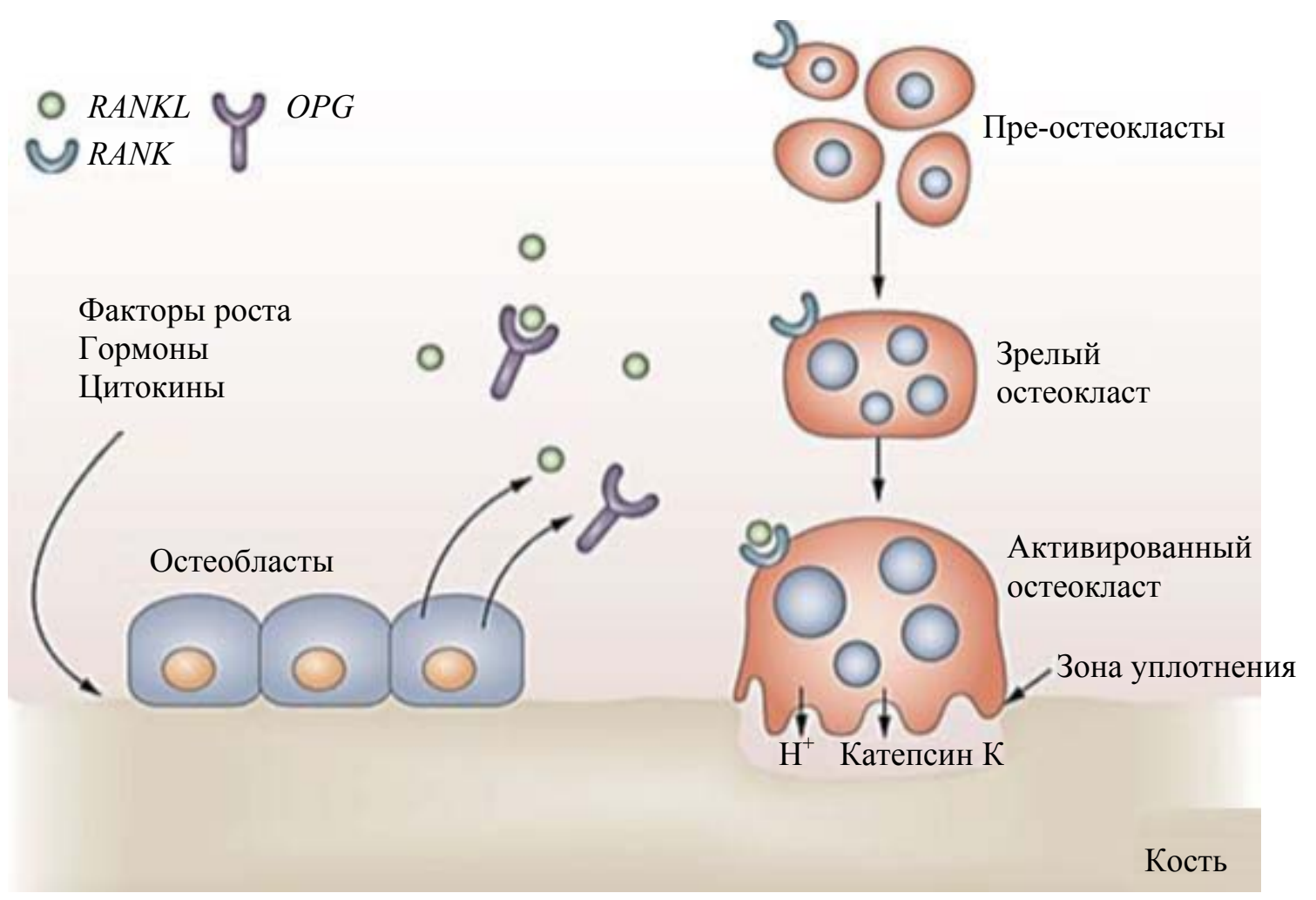

Рис. 2. Белок RANKL выражается линией остеобластов и связывается с $R A N K$ на поверхности преостеокластов и зрелых остеокластов, что приводит к увеличению костной резорбции путем увеличения дифференцировки, деятельности и выживания остеокластов. $O P G$ - это «рецептор-приманка», он также производится остеобластами, которые связываются с $R A N K L$, предотвращая связывание $R A N K$ и ингибирование резорбции костной ткани остеокластами. Это есть равновесие между $R A N K L$ и $O P G$, определяющее окончательный уровень резорбции кости [6]

Механическое регулирование, благодаря механосенсорам плазматической мембраны, происходит на уровне остеоцитов, которые переводят механические сигналы в местные биохимические реакции с целью поддержания баланса массы костной структуры; часто механический стимул определяется интрамедуллярной разностью давлений [4]; интересно провести анализ того, как такие клетки могут непосредственно сопрягаться со стимулом, заданным потоком жидкости (так называемая динамическая стимуляция потока жидкости, или $D F F S$ ), для биохимической трансдукции (вполне вероятно, что эти клетки способны отделиться) и вычислить силу механической деформации от динамических факторов потока жидкости. Эти биохимические реакции регулируют начало процесса перестройки кости и, в некотором роде, также фазу резорбции этого процесса.

Эти два механизма имеют глубокие взаимодействия, они должны быть рассмотрены в модели в виде одного параметра, т.е. они должны быть включены в переменную, принимающую во внимание физический результат этих регуляторных процессов, и делают возможным анализ популяций, вовлеченных в перестройку кости через эту единую переменную. В нескольких интересных исследованиях $[5,12]$ была предположена сильная корреляция между геометрией морфологии кости (пористостью и морфологией поверхности) и этими двумя типами настроек, и эти идеи очень полезно рассмотреть в модели. 
Таким образом, в соответствии с предложенной геометрической моделью учитываются еще два параметра: сосудистая пористость (в кортикальной костной ткани она связана с системой гаверсовых каналов, а в трабекулярной костной ткани эта пористость рассматривается в связи с пространством, расположенным между костным мозгом и трабекулярной структурой), обозначаемая $f_{v}$ и являющаяся отношением объема сосудистых пор $V_{v}$ к объему ткани $V_{t}$ порядка 1-3 мм³ [12], а именно:

$$
f_{v}=\frac{V_{v}}{V_{t}}
$$

и плотность поверхности, обозначаемая $S_{v}$, которая для пористого материала определяется отношением площади пор в интерстиции $S_{p}$ к объему ткани $V_{t}$, т.е.

$$
S_{v}=\frac{S_{p}}{V_{t}}
$$

Точно так же рассматривается доля объема костного матрикса, которая обозначается $f_{\text {mat }}$ и определяется отношением между объемом костной матрицы $V_{\text {mat }}$ и объемом ткани $V_{t}$ :

$$
f_{\text {mat }}=\frac{V_{\text {mat }}}{V_{t}}
$$

В эти соотношения не входит ни пористость, выведенная из костных лакун, ни пористость, относящаяся к костным каналам, так как оба эти параметра не участвуют в процессе перестройки.

Очень важным параметром для оценки процесса перестройки является поверхностная плотность, поскольку она относится к трехмерным свойствам структуры и способна определить в кости область, в которой будут работать остеобласты и остеокласты, а также среду, в которой остеокласты осуществляют передачу сигналов, определяющих окончательную микроструктуру.

В исследовании P. Pivonka, P.R. Buenzli et al. [12] представлено полиномиальное уравнение, взятое из работы Martin [8] (в упомянутой работе из-за большого количества экспериментальных данных автор помещает значения коэффициентов полинома (10); в этом случае $a=32,3 ; b=-93,9 ; c=-134 ; d=-101 ; e=8,28$, и вся работа ориентирована на построение и анализ такой модели), устанавливающее количественную взаимосвязь между поверхностной плотностью и сосудистой пористостью:

$$
S_{v\left(f_{v}\right)}=a f_{v}+b f_{v}^{2}+c f_{v}^{3}+d f_{v}^{4}+e f_{v}^{5} .
$$

Соотношение между поверхностной плотностью и сосудистой пористостью хорошо представлено в виде двухпараметрического графика, приведенного P. Pivonka, P.R. Buenzli et al. [12] (см. рис. 3).

Обе эти работы, P. Pivonka, P.R. Buenzli et al. [12] и Martin [8], наглядно показывают (благодаря графику на рис. 3), что все полученные экспериментальные данные соответствуют одной той же кривой $S_{v}\left(f_{v}\right)$ и делают это независимо от типа кости (с болезнью или без). Это определяет переменную $S_{v}$ (и связанную с ней кривую $\left.S_{v}\left(f_{v}\right)\right)$ в качестве внутреннего свойства кости. Таким образом, можно предположить эволюцию баланса между двумя популяциями клеток путем анализа сосудистой пористости (работу резорбции остеокластами и отложения остеобластами), и благодаря тесной взаимосвязи с поверхностной плотностью можно вычислить первую величину (пористость), анализируя вторую (плотность). 




Рис. 3. Соотношение между поверхностной плотностью и сосудистой пористостью; данные, полученные из гистологических срезов и различных типов костной ткани. Жирные черные символы - здоровая кость, нежирные символы - кость с болезнью (остеопороз, остеомаляция и несовершенный остеогенез). Кружочки - бедро человека, квадратики - гребень подвздошной кости человека, ромбики - ребро человека и треугольники - позвонок человека. Максимальная кривая (сплошная линия) для $S_{v}$ при $f_{v}=f_{v}^{*} \approx 0,37$. Пунктирные кривые были получены путем изменения параметров полинома уравнения (10) [12]

Изменения, которые можно видеть в этих двух рассматриваемых переменных, являются четким отражением согласованной и синхронной работы обоих регуляций (биохимической и механической), являющихся основными механизмами перестройки кости (восстановления и адаптации). Следовательно, система костной морфогенетической единицы становится нелинейной и периодической, но только если рассматривать ее в качестве отдельной среды, или любые предположения будут сделаны насчет пространства восстановления той же костной морфогенетической единицы.

Тогда уравнения (5) и (6) становятся справедливыми только в этом конкретном состоянии (и близко к костной морфогенетической единице), и можно далее вывести уравнение (11), а именно:

$$
\frac{d S_{v}}{d t}=\gamma_{o b} \gamma_{o c} \sum_{n=0}^{+\infty} f_{v}^{n}
$$

Здесь рассматриваем $\gamma$ как постоянный параметр, который представляет собой физическую функцию, связанную с присутствием субпопуляции линии остеокластов $\left(\gamma_{o c}\right)$ и остеобластов $\left(\gamma_{o b}\right)$, которые не вмешиваются непосредственно в процесс перестройки и самообновления. Тогда параметр $\gamma$ может быть использован для количественной оценки модели Мальтуса для популяции с непрерывной тенденцией [7]:

$$
\gamma_{x}=X_{y}^{0} e^{c t}
$$


Применяя (12) к популяции клеток-предшественников, получаем

$$
\begin{aligned}
& \gamma_{o b}=X_{o b}^{0} e^{c t}, \\
& \gamma_{o c}=X_{o c}^{0} e^{c t} .
\end{aligned}
$$

Здесь показана популяция линии клеток-предшественников $X_{y}^{0}$, и $c-$ это скорость роста вовлеченной популяции (видно, что при $c=0$ популяция остается постоянной, и это является гипотетической идеальной ситуацией; если имеются флуктуации для $c>0$ или $c<0$, то популяция, соответственно, увеличивается или угасает).

Следовательно, возможная модель для действия костной морфогенетической единицы во время перестройки кости может быть построена в предположении о справедливости уравнений (5), (6) и (11), а именно:

$$
\begin{gathered}
\frac{d X_{o s b}}{d t}=a-b \sqrt{X_{o s b(t)}}, \\
\frac{d X_{o s c}}{d t}=c \frac{X_{o s c(t)}^{2}}{K_{o s c}+X_{o s c(t)}^{2}}-d, \\
\frac{d S_{v}}{d t}=\gamma_{o b} \gamma_{o c} \sum_{n=0}^{+\infty} f_{v}^{n} .
\end{gathered}
$$

\section{ЗАКЛЮЧЕНИЕ}

Построение теоретической модели перестройки кости никогда не будет полным: многие переменные возникают по мере продолжения экспериментов в процессе исследований и анализа какой-либо новой биологической функциональности органической системы.

Предлагаемая система анализирует количественно (или по крайней мере пытается это сделать) функцию костных морфогенетических единиц в процессе перестройки кости и делает это, применяя некоторые условия:

- Костные морфогенетические единицы являются нелинейными и периодическими системами.

- Клеточные популяции учитывают принцип Гаусса как конкурентное исключение (т.е. популяции не конкурируют друг с другом на одном субстрате).

- Переменная $S_{v}$ характеризует внутреннее свойство кости и поэтому является переменной состояния рассматриваемой системы.

Таким образом, в данной работе автор пытался сделать в этой статье, это найти модель, которая будет оценивать и, если возможно, имитировать функциональность костной ткани в определенных точках, в моменты, которые непрерывно напоминают выполнить адаптацию к нагрузке (физиологической или нет) или процесс восстановления после перелома. При этом особо рассмотрены изменения, которым система костных многоклеточных единиц подвергается в случае заболеваний, имеющих большое социальное значения, таких как остеопороз.

Существуют разные математические модели для имитации перестройки кости $[2,3,12,13]$, и этому важнейшему процессу уделяется все более пристальное внимание. 
Предложенная здесь модель описывает мельчайшие подробности механизмов, учитывающие ослабление и цикличность системы, что может привести к более значительной динамике. Она осознает информационную сеть, обеспечивающую многомерную связь между всеми системами костных морфогенетических единиц и, в свою очередь, предлагает механизм, согласно которому только взаимосвязанные сетевые процессы могут имитировать и воспроизводить явление перестройки во всей его структурной сложности.

В частности, здесь можно увидеть, что:

1. Регулирование перестройки кости внутри костной морфогенетической единицы происходит благодаря структурной переменной - поверхностной плотности.

2. Стабилизация кости в рамках узких границ костной морфогенетической единицы происходит благодаря динамике вовлеченных клеточных популяций в соответствии с ростом, индуцированным от прародителей, которые экспоненциально реагируют на стартовый сигнал процесса.

3. «Ролевая игра», устанавливаемая в костной морфогенетической единице между остеобластами и остеокластами, имеет решающее значение и соответствует логике принципа Вольтерра (хищник-жертва), только если имеется равновесие в производстве клеточных популяций, как это отражено в уравнении (17).

4. Циклический характер системы тесно связан с колебаниями параметров, находящихся за пределами принципа Вольтерра $\left(\gamma_{x}\right)$, но непосредственно влияющих на соотношение роста протагонистов клеточных популяций в явлении перестройки.

Несомненно, предложенная модель допускает внесение более изощренных изменений и большего количества событий, требующих сложных вычислительных решений.

Конечно, следующим шагом может быть реализация этой модели с новой моделью сети, где костные морфогенетические единицы могут также служить в качестве мобильных узлов (или «мобильных графов») достаточно сложного вычислительного значения.

\section{СПИСОК ЛИТЕРАТУРЫ}

1. Gause G.F., Smaragdova N.P., Witt A.A. Further studies of interaction between predators and prey // Journal of Animal Ecology. - 1936. - Vol. 5, № 1. - P. 1-18. DOI: 10.2307/1087.

2. Genever P.G., Patton R.J., Fagan M. A novel mathematical model of bone remodeling cycles for trabecular bone at the cellular level // Biomech. Model. Mechanobiol. - 2012. - Vol. 11, № 7. - P. 973-982. DOI: 10.1007/s10237-011-0366-3.

3. Graham J.M., Avati B.P., Ramakrishnan P.S., Martin J.A. Toward a new spatial representing of bone remodeling // Mathematical Bioscience and Engineering. - 2012. - Vol. 9, № 2. - P. 281-295. DOI: 10.3934/mbe.2012.9.281.

4. Hu M., Tian G.W., Gibbons D.E., Jiao J., Qin Y.X. Dynamic fluid flow induced mechanobiological modulation of in situ osteocyte calcium oscillations // Archives of Biochem. Biophys. - 2015. - Vol. 579. P. 55-61. DOI: 10.1016/j.abb.2015.05.012.

5. Kostenuik P.J. Osteoprotegerin and RANKL regulate bone resorption, density, geometry and strength // Current Opinion in Pharmacology. - 2005. - Vol. 5, № 6. - P. 618-625. DOI: 10.1016/j.coph.2005.06.005.

6. Lewiecki E.M. New targets for intervention in the treatment of postmenopausal osteoporosis // Nature Review Rheumatology. - 2011. - Vol. 7, № 11. - P. 631-638. DOI: 10.1038/nrrheum.2011.130.

7. Malthus T.R. Essay on the principle of population. - London: John Murray, C Roworth, 1826.

8. Martin R.B. Porosity and specific surface of bone // Crit. Rev. Biomed. Eng. - 1984. - Vol. 10. P.179-222.

9. McMahon M.S. Bone morphogenic protein 3 signaling in the regulation of osteogenesis // Orthopedics. - 2012. - Vol. 35, No. 11. - P. 920. 
10. Parfitt A.M. The physiological and clinical significance of bone histomorphometric data // Bone histomorphometry: techniques and interpretation / ed. R.R. Recker. - Boca Raton: CRC Press, 1983. - P. 143-223.

11. Parfitt A.M. Osteonal and hemi-osteonal remodeling: the spatial and temporal framework for signal traffic in adult human bone // Journal of Cell. Biochem. - 1994. - Vol. 55, No. 3. - P. 273-286. DOI: $10.1002 / \mathrm{jcb} .240550303$.

12. Pivonka P., Buenzli P.R., Scheiner S., Hellmich C., Dunstan C.R. The influence of bone surface availability in bone remodelling - A mathematical model including coupled geometrical and biomechanical regulations of bone cells // Engineering Structures. - 2013. - Vol. 47. - P. 134-147. DOI: 10.1016/j.engstruct.2012.09.006.

13. Robling A.G., Castillo A.B., Turner C.H. Biomechanical and molecular regualtion of bone remodeling // Annu. Rev. Biomed. Eng. - 2006. - Vol. 8. - P. 455-498. DOI: 10.1146/annurev.bioeng.8.061505.095721.

14. Volterra V. Fluctuations in the abundance of a species considered mathematically // Nature. - 1926. Vol. 118, № 2972. - P. 558-560. DOI: 10.1038/118558a0.

15. Volterra V. Variazioni e fluttuazioni del numero d'individui in specie di animali conviventi [Электронный ресурс]. - URL: www.liberliber.it/mediateca/libri/v/volterra/variazioni_e_fluttuazioni/pdf/ volterra_variazioni_e_fluttuazioni.pdf).

\section{THEORETICAL MODEL FOR BONE REMODELLING}

\section{A. Nutini (Lucca, Italy)}

The remodelling is a complex process essential for the physiological functioning of the bone tissue. In this paper, the author tries to create a theoretical model for this process considering both biomechanical and biochemical regulations. Bone remodelling, therefore, realizes a complex network that in turn stimulates the conformational changes required for both the health of the bone tissue and the repair processes. After briefly analyzing the physiology of the bone remodelling phenomenon, the author considers the mathematical development of idea of Vito Volterra on the ecological relations between predator and prey populations adjusting it thanks to a strict analogy to the dynamics of cell populations which are the protagonists in the bone remodelling phenomenon. Considering the oscillatory nature of the phenomenon in the environment of the bone morphogenetic units, the model is completed by a strong correlation between the geometry of the bone morphology (porosity and surface morphology) and the two regulations above. A new state variable is also added to a differential equation that considers subpopulations of both osteoclasts and osteoblasts that do not directly intervene in the remodelling process (the precursors of the cell lines) and process of self-renewing. In this way, the proposed model is conducted by three differential equations that describe the tissue's state (in health or pathological situations) and the work of remodelling in action.

Key words: theoretical model, remodelling, osteoclasts, osteoblasts, bone morphogenetic unit, prey/predator model, Vito Volterra theory, bone tissue. 